\title{
Study on the Extraction Rate of Morphine Before and After Hair Grinding
}

\author{
Xiao Ruisen*, Huang Ping, Wang Hong, Li Jinwei \\ Department of Technology, Tianjin Ankang Hospital, Tianjing, China \\ Email address: \\ xruisen@163.com(Xiao Ruisen) \\ ${ }^{*}$ Corresponding author
}

To cite this article:

Xiao Ruisen, Huang Ping, Wang Hong, Li Jinwei. Study on the Extraction Rate of Morphine Before and After Hair Grinding. Journal of Drug Design and Medicinal Chemistry. Vol. 4, No. 1, 2018, pp. 1-4. doi: 10.11648/j.jddmc.20180401.11

Received: May 3, 2018; Accepted: May 30, 2018; Published: June 19, 2018

\begin{abstract}
: 1500 morphine-absorbed hair was collected, and the hair was ground with a quick grinder to extract the morphine component of the hair. The morphine was qualitatively detected by gas chromatography/mass spectrometry (GC/MS). The test results were processed by statistical software and the hair was evaluated. Morphine positive tests results. A total of 1500 morphine users were found to have 1465 morphine-positive (97.7\%) in the hair-dried group and 998 morphine-positive (66.5\%) in the unpolished hair group. The results were statistically significant $(\mathrm{P}<0.005)$. In the analysis of the influencing factors of the testing method, the sample collected by the sex, age, and hair collection location of the drug addicts had no effect on the test results $(\mathrm{P}>0.05)$; the chemical dyeing, regardless of whether the hair was ground or not, showed a significant decrease in morphine positive detection. The test results have an effect $(\mathrm{P}<0.05)$. The results of the study showed that morphine was extracted from ground hair and the positive detection of morphine was significantly improved, with a positive rate of $97.67 \%$. This prevented the missed detection in the drug addiction certification work and made the certification test more scientific and accurate.
\end{abstract}

Keywords: Hair Grinding, Extraction Rate, Morphine, Addiction Identification

\section{Introduction}

The drug problem is a scourge for individuals, families, organizations and societies. According to the latest statistics, as of December 2015, China registered a total of 3.34 million registered drug addicts [1]. On April 1, 2011, the Ministry of Public Security of the People's Republic of China and the Ministry of Health of the People's Republic of China jointly promulgated the criteria for the determination of addiction to drug addicts: 1 . The human biological sample test proved that it contained drug components. 2 . There is evidence to prove that he used drugs and he was found to be drug addiction [2]. As the drug is metabolized in the blood and urine, samples that are greater than 72 hours, the qualitative detection of the drug is prone to negative, resulting in missed tests for drug addiction certification. Human hair, as a special carrier, is easy to collect, easy to store, and can retain drug information for a long period of time in drug detection. It reflects changes in the time range of drug use and drug use. In recent years, hair drug analysis has become a forensic science and clinical analysis of poisons. Research hotspots [3-6]. Current research results show that the extraction rate of morphine in unmilled hair is low [7-12]. In view of the low detection rate of morphine in unmilled hair, we conducted a study on the extraction of morphine from the ground hair, trying to increase the positive detection rate of morphine in the hair of morphine suspects, and more accurately determine drug addiction.

\section{Research Objects and Methods}

\subsection{Research Object}

Subjects selected from January 2015 to May 2016 were detained at drug abuse sites in Tianjin and Hebei Provinces according to the following criteria: (1) consuming morphine; (2) aged 20 to 50 years; (3) no serious endangerment of drug addicts after initial inspection Diseases of life; (4) Actively cooperate with leaving a sample. At the same time excluded: (1) smoking drugs other than morphine; (2) taking antibiotic 
tranquilizers within one week; (3) suicidal self-mutilation with severe violence; (4) HIV patients. A total of 1500 participants were selected for the study, and the participants obtained written informed consent.

\subsection{Grouping}

The 1500 morphine-absorbed hair was used as the experimental group, and 1500 unpolished hair was used as the control group.

\subsection{Hair Sample Collection and Cleaning}

Two 50-mg portions of a $1 \mathrm{~cm}$ hair sample on the hair root were collected, $50 \mathrm{mg}$ of the ground hair was incorporated into the experimental group, and $50 \mathrm{mg}$ of un-ground hair was placed in the control group.

Add $0.1 \%$ sodium dodecyl sulfonate, $0.1 \%$ detergent, water and acetone successively and dry. The experimental group was grinded with 360s and $50 \mathrm{~Hz}$, and the control group was cut into $5 \mathrm{~mm}$ segments.

\subsection{Grinding}

$2.5 \mathrm{~mm}$ nickel-cadmium alloy steel balls are vortexes together with the hair to destroy the hair fibers, increase the surface area of the hair, and increase the release efficiency of morphine. According to the results of the pre-test, the best value for the hair to be fully ground is $360 \mathrm{~s}, 50 \mathrm{~Hz}$.

\subsection{Digestion and Extraction}

The $0.5 \mathrm{~mol} / \mathrm{L} \mathrm{HCL}$ solution was soaked overnight at $37^{\circ} \mathrm{C}$., extracted with a chloroform:isopropyl alcohol (9:1) extract, and the organic layer was evaporated at $60^{\circ} \mathrm{C}$.

\subsection{Derivative and Detection}

After derivatization with MSTFA at $60^{\circ} \mathrm{C}$ for $30 \mathrm{~min}$, samples were tested.

Gas chromatography-mass spectrometry (GC/MS), determination conditions--Column HP-5MS capillary column, column temperature $100^{\circ} \mathrm{C}$ for $1.5 \mathrm{~min}$, temperature increase to $280^{\circ} \mathrm{C}$ at $25^{\circ} \mathrm{C} / \mathrm{min}$, hold for $15 \mathrm{~min}$, carrier gas: helium, purity
99.999\%, flow rate: $1.0 \mathrm{~mL} / \mathrm{min}$, relative voltage $0.1 \mathrm{eV}$.

\subsection{Qualitative Result Determination}

The peak retention time (RT) of the target in the sample with the blank addition standard and the characteristic fragment ion of the mass spectrum were respectively compared to satisfy the target ion $M / Z 236$, reference ions $\mathrm{M} / \mathrm{Z414}$, M/Z429, and the retention time was $11.635 \mathrm{~min}$. When two conditions are satisfied, it is determined that the corresponding substance is detected, otherwise, it is determined that it is not detected.

\subsection{Analysis of Factors Affecting Detection Methods}

The effects of the test results on the subjects' gender, age, hair collection site, staining and non-staining factors were analyzed.

\subsection{Statistical Methods}

All data entry computers were processed with SPSS 21.0 statistical software. The comparison between the two groups was based on data distribution using t-test, multiple groups of data were compared using analysis of variance, multivariate analysis was used as the dependent variable, and multivariate logistic regression analysis was used to complete the analysis. (using default parameters), $\mathrm{P}<0.05$ was considered statistically significant.

\section{Results}

\subsection{Comparison of the Number of Morphine Positive Cases Before and After Hair Grinding}

Among 1500 patients who took morphine, positive results were detected in Table 1. The result of the1465 were morphine positive in the hair polishing group, and the positive rate was $97.67 \%$, In the unground group, 998 cases of morphine were positive, and the positive rate was $66.53 \%$. Statistical analysis $\mathrm{X} 2=467, \mathrm{P}<0.005$ There were significant differences between the two detection methods. The detection rate of morphine after grinding was higher.

Table 1. Comparison of morphine positive detection before and after hair grinding.

\begin{tabular}{lllll}
\hline Grinding & & & & \\
\hline Grouping & & Morphine positive (n) & Morphine-negative (n) & Total (n) \\
\hline \multirow{3}{*}{ Unground Total (n) } & Morphine positive (n) & 998 & 0 & 998 \\
& Morphine-negative (n) & 467 & 35 & 502 \\
\\
\cline { 2 - 5 }
\end{tabular}

\subsection{Analysis of Influencing Factors of Detection Methods}

Among 1500 morphine smokers, the basic conditions affecting the test are described in Table 2, the factors affecting the test result are described in Table 3. The detection of morphine was significantly higher in aged hairs aged 20-30 years, 31-40 years, and 41-50 years, and morphine was detected in unmilled hair. There was no statistically significant difference in the morphine rate between the three groups of hair in the grinding group $(\mathrm{P}>0.05)$. The statistical analysis of morphine in the unground group ( $\mathrm{Sig}=0.28, \mathrm{P}>0.05$ ) was not statistically significant. At the site of hair collection, 134 cases were collected at the top of the hair, 187 cases were collected from the left iliac crest, 159 cases were taken from the right iliac crest, and 132 cases of morphine were collected from the occipital area. The positive detection of morphine was $94.7 \%$, $97.9 \%, 98.7 \%$, and $99 \%$ respectively. The morphine was detected when the parts were not ground. There was no 
statistical difference in the morphine detection in the ground hair of the four collection sites ( $\mathrm{Sig}=0.09, \mathrm{P}>0.05$ ). No statistical analysis was performed in the unground group morphine (Sig $=0.07, \mathrm{P}>0.05)$. Academic significance in terms of gender, positive detections of morphine in 980 males and 520 females with ground hair were $99.9 \%$ and $93.7 \%$, respectively, which were higher than those of morphine when unpolished in various parts. There was no statistical significance in the detection of morphine in the ground hair of the men's and women's hair ( $\mathrm{Sig}=0.49, \mathrm{P}>0.05)$. No statistically significant difference was found in the morphine of the unground group ( $\mathrm{Sig}=0.52, \mathrm{P}>0.05$ ). There were 231 hair dyes and 1269 unstained hairs. The positive morphine in the ground hair was detected in $84.4 \%$, and in the unstained hair 1269 in the ground hair, the morphine was detected as $100 \%$. The statistical analysis of morphine in the ground and non-stained hair groups was statistically significant ( $\mathrm{Sig}=$ $0.008, \mathrm{P}<0.05)$. Statistical analysis was performed in the unground group with morphine $(\mathrm{Sig}=0.002, \mathrm{P}<0.05)$.

Table 2. Basic conditions of impact testing.

\begin{tabular}{|c|c|c|c|c|c|}
\hline \multirow{2}{*}{ Influencing Factors } & \multirow{2}{*}{ Number of cases (n) } & \multicolumn{2}{|l|}{ Grinding } & \multicolumn{2}{|l|}{ Unground } \\
\hline & & Morphine positive (n) & Morphine negative (n) & Morphine positive (n) & Morphine negative (n) \\
\hline 20-30 years old & 623 & 601 & 22 & 442 & 181 \\
\hline $31-40$ years old & 520 & 510 & 10 & 332 & 188 \\
\hline $41-50$ years old & 357 & 354 & 3 & 224 & 133 \\
\hline Male & 980 & 979 & 1 & 848 & 132 \\
\hline Female & 520 & 487 & 33 & 148 & 372 \\
\hline Top & 360 & 341 & 19 & 271 & 99 \\
\hline Right & 392 & 387 & 5 & 252 & 140 \\
\hline Pillow & 408 & 404 & 4 & 253 & 155 \\
\hline Dyeing & 231 & 195 & 36 & 39 & 192 \\
\hline
\end{tabular}

Table 3. Factors affecting test results.

\begin{tabular}{|c|c|c|c|c|c|c|c|c|c|c|}
\hline \multirow[t]{2}{*}{ Grouping } & \multicolumn{2}{|c|}{ Grinding samples } & \multicolumn{8}{|c|}{ Unground sample } \\
\hline & B & "S. E," & Wals & df & Sig. & B & "S. E," & Wals & df & Sig. \\
\hline age & 0.06 & 0.03 & 4.3 & 1 & 0.04 & -0.03 & 0.01 & 8.6 & 1 & 0 \\
\hline Gender (for fem ale reference) & -1.06 & 1.52 & 0.49 & 1 & 0.49 & 2.43 & 0.17 & 217.16 & 1 & 0 \\
\hline Staining (with no staining as a reference) & -20.19 & 1095.33 & 0 & 1 & 0.99 & -1.54 & 0.23 & 44.63 & 1 & 0 \\
\hline Top (reference site) & & & 5.83 & 3 & 0.12 & & & 58.98 & 3 & 0 \\
\hline Left & 0.79 & 0.46 & 2.99 & 1 & 0.08 & -0.91 & 0.23 & 16.15 & 1 & 0 \\
\hline Right & 0.96 & 0.55 & 3.04 & 1 & 0.08 & -1.36 & 0.22 & 37.63 & 1 & 0 \\
\hline Constant & 19.57 & 1095.33 & 0 & 1 & 0.99 & 1.41 & 0.37 & 14.42 & 1 & 0 \\
\hline
\end{tabular}

\section{Discussion}

In this study, qualitative detection of morphine in hair was used as an indicator. Different ages, sex, different parts of the head, and dyed unstained hair were used as research factors to investigate the effect of morphine release before and after grinding. Studies have shown that the positive detection rate of morphine after grinding hair is significantly higher than the positive rate of non-abrasive detection.

Hair is a complex tissue. According to the "embedded model" theory [13,14], drugs that enter the bloodstream enter the root region of the hair during hair growth. After the hair grows out of the scalp, the drugs form a band in the hair, with a certain concentration. Embedded in keratin in hairs. To detect these drug ingredients, they first need to be released and released. In the past, the use of alkaline digestion, acid digestion, enzyme digestion, ultrasound digestion [15-19] method to release morphine detection rate is low. This study used physical methods to destroy the hair fibers, increase the surface area of the hair, and release morphine from the embedded keratin. The detection rate of morphine positive rate was significantly improved. The experimental results showed that the positive morphine detection rate was $97.7 \%$ after hair was ground, and the positive morphine detection rate was $66.5 \%$.

The hair collected in the study was affected by gender, age, and hair collection site, and hair thickness, softness, color, and texture were different [20] and may affect the test results. However, studies have found that the above factors have no effect on the release of morphine in the hair before and after grinding. May be related to the protection of the hair mechanism by drugs into the hair [21, 22].

After chemical staining, the detection rate of morphine before grinding was only $16.88 \%$, and the detection rate of morphine after grinding was $84.41 \%$, which was lower than the detection rate of undyed hair morphine. This was associated with Meng Pinjia and He Hongyuan et al. [23] The conclusions of the study are consistent. This is because the hair dye is to soften and expand the hair of the artificial pigment particles through the "alkaline" and penetrate into the semi-contact area through the easy contact area of the hair. When these particles form a large molecule, they are stabilized in the semi-contact area to form a permanent effect. This effect makes the drug in the hair less likely to release, resulting in a low morphine detection rate. Research shows, the positive rate 
of 520 female morphine smokers was low, mainly related to their long-term hair dyeing.

\section{Conclusion}

In previous hair drug analysis, the main reason for the low rate of morphine detection in hair was the difficulty in extracting drug from hair. The current work is through physical grinding, destroying the hair fiber structure and releasing morphine embedded in keratin. The detection rate is increased to $99.7 \%$. There was no correlation between the release of morphine in the hair and the sex, age, and location of the subjects. However, it is related to the dyeing of hair and has an effect on the extraction of morphine. In the study, it was found that the extraction of morphine in dyed hair was lower than that of normal hair, and the influence of this factor must be fully taken into account in actual tests. Preliminary studies have shown that the use of ground hair for the detection of morphine can increase the detection rate of morphine suspects, which is helpful for the certification of addiction.

\section{References}

[1] "Approved Measures for Drug Addiction" Ministry of Public Security of the People's Republic of China and Order No. 115 of the Ministry of Health of the People's Republic of China April 1, 2011.

[2] Tang. Y. Drug dependence and prevention of recurrence. Drug dependence on China, 1997, 6 (2): 73-76.

[3] Men GP in Jia, Zhang Q i, X u ying. hair drug analysis. Beijing, Chinese peoples public security university press, 2001: 165-178.

[4] A. Orfanidis; O. Mastrogianni; A. Koukou; G. Psarros; H. Gika; G. Theodoridis; N. Raikos. A GC-MS method for the detection and quantitation of ten major drugs of abuse in human hair samples. Journal of Chromatography B 2016- Elsevierjournal 10. 1016/j. jchromb. 2016. 11. 011.

[5] Sun Ying-Ying; Xiang Ping; Shen Min. Simultaneous determination of 11 opiates in hair by liquid chromatography-tandem mass spectrometry [J]. Yaoxue Xuebao 2012-12 PubMed.

[6] Grabenauer Megan; Bynum Nichole D; Moore Katherine N; White Robert M; Mitchell John M; Hayes Eugene D; Flegel Ronald. Detection and quantification of codeine-6-glucuronide, hydromorphone-3-glucuronide, oxymorphone-3-glucuronide, morphine 3-glucuronide and morphine-6-glucuronide in human hair from opioid users by LC-MS-MS. Grabenauer Megan Journal of analytical toxicology 2017- PubMed 10. 1093/jat/bkx086

[7] Concheiro M, Simoes SM, Quintela O, de Castro A, Dias MJ, Cruz A, Lopez-Rivadulla M. Fast LC-MS/MS method for the determination of amphetamine, methamphetamine, MDA, MDMA, MDEA, MBDB and PMA in urine. Forensic science international. 2007, 171:44-51.

[8] He Y, J. KY. J Chromatogr A. 2006, 1133:50-55.

[9] Musshoff F, Arrey T, Strupat K. Determination of cocaine, cocaine metabolites and cannabinoids in single hairs by MALDI Fourier transform mass spectrometry--preliminary results. Drug testing and analysis 2013, 5 (5): 361-365.

[10] Maria N, Claudia T, Lucia B. Cross-reactivities of 41new amphetamine designer drugs to EMIT immunoassays. Forensic Toxicology, 2012, 31 (1): 133-137.

[11] Cheong JC, Suh S, Ko BJ, Lee JI, Kim JY, Suh YJ, In MK. Screening method for the detection of methamphetamine in hair using fluorescence polarization immunoassay. Journal of analytical toxicology, 2013, 37 (4): 217-221.

[12] Shao. M. Try to talk about the cause of drug abuse. The rule of law and society, 2005, 3:250-251.

[13] Li. X, Yuan. C, Wen. H. The drug addicts and personal integrity. Chinese journal of drug dependence, 2005, 14 (3): 226-228.

[14] S. Nikfar; M. Jaberidoost. Hair. 2014 Elsevier 10. 1016/B978-0-12-386454-3. 00610-2.

[15] Nishida M. Yashiki M. Namera A. et al. J Chromatogr B. 2006, 842:106-110.

[16] Gentili S. Cornetta M. Macchia T. J Chromatogr B. 2004, 801:289-296.

[17] Polettini A. Stramesi C. Vignali C. et al. Foren Sci Int. 1997, 84:259-269.

[18] Cordero R. Paterson S. Chromatogr B. 2007, 850:423-431.

[19] Cappelle Delphine; De Keukeleire Steven; Neels Hugo; Been Frederic; De Doncker Mireille; Dom Geert; Crunelle Cleo L; Covaci Adrian; van Nuijs Alexander L N. Keratinous matrices for the assessment of drugs of abuse consumption: A correlation study between hair and nails. Drug testing and analysis [J]. 2018- PubMed 10. 1002/dta. 2356.

[20] Cao Xiaoci, DING Penglin, ZHONG Mingtian et al. Correlation between TCM syndromes and liver function indexes in different product addicts [J]. Chinese Journal of Drug Dependence, 2016, 25(2): 182-185.

[21] Xie P, Wang TJ, Yin G. Hair testing drug abuse technology. Drug dependence on China, 2014, 20 (1): 120-126

[22] Klima Miriam; Altenburger Markus J; Kempf Jürgen; Auwärter Volker; Neukamm Merja A. Determination of medicinal and illicit drugs in post mortem dental hard tissues and comparison with analytical results for body fluids and hair samples. Forensic science international. 2016- PubMed 10. 1016/j. forsciint. 2016. 02. 021.

[23] Meng. P, He. H, Zhu. D. Analysis and explanation of drugs and their metabolites in drug abusers' hair. Journal of pharmaceutical analysis, 2008, 5:709-714. 